\begin{tabular}{|c|l|}
\hline Title & Three Dimensional Cellular A utomaton Model of Segregation of Granular Materials in a Rotating Cylinder \\
\hline Author(s) & Y anagita, Tatsuo \\
\hline Citation & $\begin{array}{l}\text { PHY SICAL REV IEW LETTERS, 82(17), 3488-3491 } \\
\text { https://doi.org/L0.1103/PhySRevLett.82.3488 }\end{array}$ \\
\hline Issue Date & 1999-04 26 \\
\hline Doc URL & http://hdl.handle.net/2115/5752 \\
\hline Rights & Copyright $\odot 1999$ A merican Physical Society \\
\hline Type & article \\
\hline File Information & PRL82-17.pdf \\
\hline
\end{tabular}

Instructions for use 


\title{
Three-Dimensional Cellular Automaton Model of Segregation of Granular Materials in a Rotating Cylinder
}

\author{
Tatsuo Yanagita* \\ Research Institute for Electronic Science, Hokkaido University, Sapporo, Hokkaido 060, Japan
}

(Received 17 August 1998)

\begin{abstract}
Binary mixtures of granular materials often exhibit segregation along the axis of a horizontal rotating cylinder. It is well known that segregation occurs radially at first, forming a buried core of one component. Later, alternate banding starts to form. This is axial segregation. There is no threedimensional model that is capable of depicting transition from radial to axial segregation. We propose a cellular automaton model in which particles slide on the surface, and show that this model successfully reproduces these segregation processes. [S0031-9007(99)09044-4]
\end{abstract}

PACS numbers: 64.75.+g, 81.05.Rm, 83.20.Jp

Heterogeneous mixtures of granular media tend to segregate when they are sheared, shaken, or piled [15]. Horizontal drum experiments show this clearly. An initially homogenous binary mixture of granular media subjected to rotation in a horizontal cylinder separates into alternating bands of the two species arranged along the axis of the cylinder [6-9]. Axial segregation was first observed by Oyama [6] (most of Oyama's work is written in Japanese but his work was translated and summarized in [10]). All granular mixtures that axially segregate in horizontally rotated cylinders also display radial segregation $[6,7,11]$. For a mixture of different sized particles, smaller particles form a radial core before axial segregation occurs.

There are many studies of the segregation in a twodimensional rotating-drum mixer [12,13]; however, to my knowledge, there-dimensional models which describe axial segregation are missing. The aim of this Letter is to introduce a three-dimensional cellular automaton (CA) model that is capable of reproducing both radial and axial segregations (Fig. 1).

Physicists have recently turned their attention to these segregation phenomena $[14,15]$. It was believed that axial segregation is driven by a sorting process occurring at the flowing surface. The dynamic angle of repose, defined as the angle at which the surface streams down for a given rotation frequency, is a function of the local composition of the mixture. Indeed, after axial segregation has been completed, one can easily observe that modulation of the dynamic angle of repose is associated with bands of corresponding constituents. These observations have lead to models of surface-slope (the undulations of the dynamic angle of repose) driven axial segregation which simply ignore the bulk motion of the particles.

Magnetic resonance imaging (MRI) measurements of internal structure provide additional information that is useful for constructing a model. Hill, Caprihan, and Kakakios [15] have shown that the axial bands are connected through the radial core. Indeed, it was suggested that banding patterns might be better viewed as undula- tions in the thickness of the core which break through the surface. On the other hand, Nakagawa et al. [16] show completely segregated axial bands. The mechanisms are not yet understood and there may be different ways to reproduce axial segregation. Our model here is based on the assumption that the axial segregation is driven by the difference in the dynamic angle of repose. We can neglect the motion of particles in the bulk to reproduce the axial segregation. (There might be a situation in which we cannot neglect the motion in the bulk. The drum mixer fully filled with particles is one of the examples. In this case, motion in the bulk is much more important for the segregation [17].)

The large number of particles and long time scale make discrete particle simulation (discrete element method, for (a)

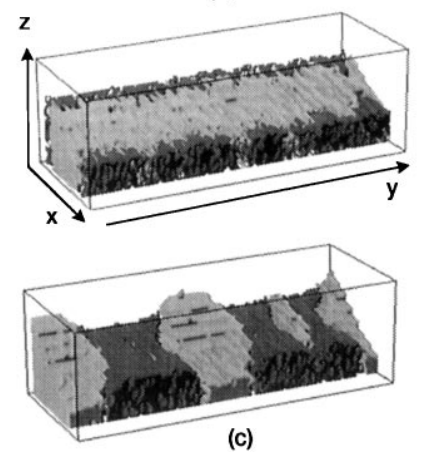

(b)
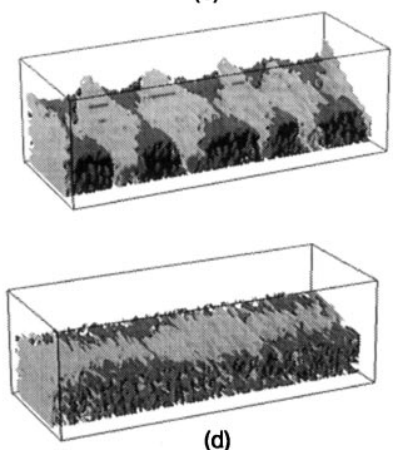

FIG. 1. Isosurfaces at $\sigma(x, y, z)=1,2$ (the boundaries between the $A$ and $B$ particles) are plotted by the different shades; the lighter shade is the particle $A$ boundary. (a) $t=100$, the snapshot after the radial segregation. The sandwich structure is clearly shown. It starts from well-mixed initial conditions. (b) $t=1000$, the $A$ and $B$ particles are axially segregated. (c) $t=10000$, after long time evolution, the above narrow band merged and coarsened bands appeared. We can see the banding pattern with this large aspect ratio simulation. $f_{A A}=0.4, f_{A B}=0.44, f_{B B}=1.0, N_{x}=10, N_{y}=200$, and $N_{z}=40$. (d) We have observed undulations of the radial core. If the $f_{B B}$ is smaller, these undulations do not reach the front wall $x=0$. $t=10000, f_{A A}=0.4, f_{A B}=0.44, f_{B B}=0.7$, $N_{x}=10, N_{y}=200$, and $N_{z}=40$. 
example) difficult. Furthermore, it is sometimes not easy to completely understand the phenomenology of the segregation of particulate media by using direct simulations. In order to understand the segregation process for large systems over a long time, a phenomenological model can provide good insights. Our three-dimensional CA model enables one to investigate the large scale spatiotemporal behavior of the segregation process of granular media in a rotating cylinder.

A cellular automaton is a dynamical system with discrete space, time and state variables [18]. Here, we use a three-dimensional square lattice $(x, y, z)$, where $y$ and $z$ are the axial and vertical directions. Each site has a discrete field variable $\sigma(x, y, z)$ which takes a value from $\{0,1,2\}$ corresponding to empty, occupied by $A$, or occupied by $B$ particles, respectively.

Following Zik et al. [14], we assume that segregation occurs entirely due to particle motion on the free surface, and that the friction plays an essential part in the segregation process. (Zik also noted that the shape of the free surface of the system plays an important role in the segregation, and cannot simply be considered as a flat slope. Our model reproduces this S-shaped surface by simulations.) This model can be easily generalized to study a mixture of grains of different sizes. Indeed, a somewhat similar model has been developed to explain the stratification mechanism [19]. The dynamics of the field variable involves the following two steps: the sliding motion of particle on the surface and the rotation of the container. These two physical processes are carried out as follows: (1) We choose a particle on the surface $\sigma((x, y, h(x, y))$ randomly, where $h(x, y)$ is the height from the bottom to the surface at the position $(x, y)$ (note that all of the particles are packed in the $z$ direction due to gravity). $h(x, y)=\sum_{z=1}^{N_{z}} \Theta(\sigma(x, y, z))$, where $\Theta$ is the Heaviside function. (2) The selected particle slides down due to gravity. The next position of the particle is chosen from the neighboring sites $(x+\Delta x, y+\Delta y, h(x+\Delta x, y+\Delta y)+1), \quad$ where $\Delta x$ and $\Delta y$ are random variables taking the values of $\Delta x=\{0,1\}$ and $\Delta y=\{-1,0,1\}$ with uniform probability. (3) The sliding motion above is accepted if the height difference between the two positions $\Delta h=h(x, y)-h(x+\Delta x, y+\Delta y)$ is larger than the "effective" friction $F$ acting on the particle. [Let us consider the granular materials consisting of two types of grains $A$ and $B$ which differ only in their frictional properties. Suppose type- $A$ grains are smooth (for example, glass beads) and type- $B$ grains are rough (for example, sand); their difference is modeled by different frictional properties, among them, $f_{A A}, f_{A B}$, and $f_{B B}$. These frictional properties determine the critical height to slide. The smooth grains $A$ have a smaller angle of repose and hence a smaller frictional property.] The effective friction $F$ is a sum of the local friction which comes from the interactions of adjacent particles. (The friction $F$ gives us the transition rule for the sliding event.) In experiments, the local interaction depends on the surface characteristics, the size, the mass of the particles, and rotational frequency. Here, we use the following simple interaction rule: $F=$ $\sum_{\delta x=0,-1} \sum_{\delta y=-1,0,1} \sum_{\delta z=0,-1} f[\sigma(x, y, h(x, y)) \sigma(x+$ $\delta x, y+\delta y, h(x, y)+\delta z)]$, where $f$ is a local map defined as $f:(0,1,2,4) \rightarrow\left(0, f_{A A}, f_{A B}, f_{B B}\right)$, with $f_{A A}$, $f_{A B}$, and $f_{B B}$ being adjustable parameters. (The combination of two adjacent particles can be expressed by the multiplication of the state variables.) (4) The above three procedures are applied $r$ times repeatedly (the parameter $r$ is related to the inverse of the rotational frequency $\omega=1 / r$ of the experiment). (When $\omega$ is smaller, the profile of the dynamic angle of repose changes from an $\mathrm{S}$ shape to a straight line.) The rotation of the container is simply introduced as follows (see Fig. 2): The whole region is divided into two parts, the lower $(L)$ and higher $(H)$ sides, by the vertical plane $x=N_{x} / 2$. Slide the $H$ side upward and the $L$ side downward by one cell, and then turn over the bottom layer of the $L$ side and attach it to the bottom of the $H$ side. Note that we have a stepwise height difference at the interface $x=N_{x} / 2$. Initially, the particles at $x=N_{x} / 2$ move down by the sliding procedure. However, this collapse propagates and we have a smooth dynamic angle of repose after a series of sliding procedures. This completes one cycle of the dynamics.

We use fixed boundary conditions at $y=0, y=N_{y}$; we set $B$ particles at $y=-1$ and $y=N_{y}+1$ side walls. The particles at $x=0$ cannot move in the $x$ direction, while they can move downward by the rotation of the container. We used mainly the following values for the parameters: the local effective friction $f_{A A}=0.4$, $f_{A B}=0.44, f_{B B}=1.0$, the rotational speed $r=30$, and the mixing ratio of two particles $\lambda=0.5$.

Starting with a homogeneous mixture, we first observe a sandwichlike structure as shown in Fig. 1(a). Since the container is rectangular and the depth of the flowing surface is 1, this sandwich pattern corresponds to the core structure in experiments. This radial segregation is
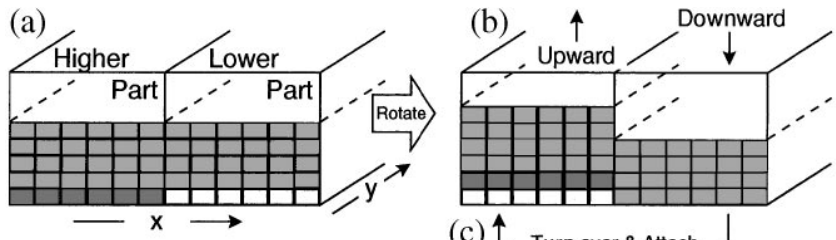

FIG. 2. Schematic explanation of the rotation procedure. (a) The whole region is divided into two parts, the lower $(L)$ and the higher $(H)$ sides. (b) Shows that the $H$ side slid upward and the $L$ side slid downward by one cell. (c) The bottom layer of the $L$ side is turned over and attached to the bottom of the $H$ side. 
achieved in 100 time steps in the simulation. The profile of the free surface shows an $\mathrm{S}$ shape (Fig. 3).

The S-shaped profile observed in our model means that the dynamic angle of repose does not converge to the static angle. For smaller rotational speeds, the profile of the slope is a straight line. The radial segregation can be explained as follows: The $A$ particles have a smaller angle of repose than the mixture of $A$ and $B$ particles (corresponding to the following condition: $f_{A A}<f_{A B}<$ $\left.f_{B B}\right)$. As a result, particles $A$ on the surface of the mixture can easily reach the end of the free surface (i.e., $x=N_{x}$ ), and the particles are again carried back to the top by the rotation of the cylinder. On the other hand, $B$ particles often remain in their own positions since the repose angle is larger than the mixture. Once the $B$ particles rotate in the central region, the angle of free surface becomes larger than that around the cylinder; thus $A$ particles always pass through the central region quickly, and therefore a quasistationary core of a $B$ particle is formed.

After radial segregation is completed, the sandwich structure becomes unstable. The weak undulations of the radially segregated structure appear, as shown in Fig. 1(b). These undulations in the core develop into bands that reach the front $x=0$ wall [Fig. 1(c)]. In order to investigate the mechanism of axial segregation, we have measured the gradient of the surface profile $\nabla h(x, y)$ and the time averaged flux of $A$ and $B$ particles. Figure 4(a) clearly shows that the $y$ component of the gradient $[\nabla h(x, y)]_{y}$ of the higher part $\left(y<N_{y} / 2\right)$ is not symmetric with that of the lower part $\left(y>N_{y} / 2\right)$. This asymmetry of the gradient field yields the mean flux of the $B$ particle, leading to the axial segregation [see Fig. 4(c)]. The flux of the $B$ particle is almost proportional to the gradient of the surface profile; however, the flux of the A particle does not follow the gradient [Fig. 4(b)]. This can be explained as follows: Consider an $A$ particle at $(x, y)$ on the surface, the possible movement is slipping down to $(x+\Delta x, y+1)$, where $\Delta x=-1,0,1$. If the entire height difference $h(x+\Delta x, y+1)-h(x, y)$ is

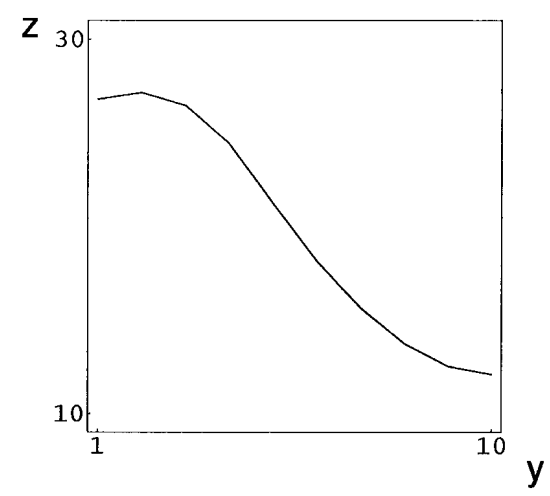

FIG. 3. Side view of the surface $h(x, y)$ at $x=40$. The Sshaped profile is clearly shown, but the plateau of the higher part $(y \sim 1)$ is wider than that of the lower part $(y \sim 10)$. larger than a critical value, the particle can slide in any possible direction; the motion along the axial direction is Brownian-like. Indeed, there is no mean flux of the $A$ particle at the higher mixing ratio position, where $(\nabla h)_{y}$ is enough to transfer the $A$ particle in any possible direction. Near the boundaries at $y=1$ and 10, the gradient $(\nabla h)_{y}$ is not enough to slide the particles; the $x$ component of the gradient $(\nabla h)_{x}$ affects the motion along $x$ direction. Because the slope of the higher part has a wider plateau [where $(\nabla h)_{y}$ is small] than that of the lower part, a larger flux of the $A$ particle near $y=1$ is observed. Both spatial patterns of the mean fluxes for $A$ and $B$ particles lead to axial segregation.

Through extensive simulations by changing parameters, we have found that, with smaller $f_{B B}$, the undulations of the core buried by the $A$ particles could not reach the front wall [Fig. 1(d)]. These situations agree with recent MRI experiments [15]. Furthermore, the axial segregation slows down and the wavelength of the undulation increases with decreasing $f_{B B}$ while $0.5<f_{B B}<0.7$. When $f_{A B}$ increases to $f_{B B}=1.0$, the axial segregation is also weakened and, if $f_{A B}>f_{B B}$, no radial and axial segregations are observed. While $0.2<f_{A B}<0.8$, $f_{A A}=0.4$, and $f_{B B}=1.0$, complete axial segregation is observed.

Our model enables us to investigate a large space and time scale simulation, such as the coarsening process of
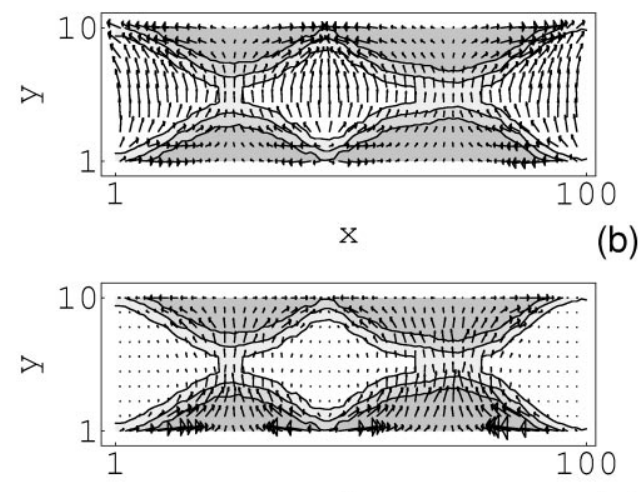

$\mathrm{x}$

(c)

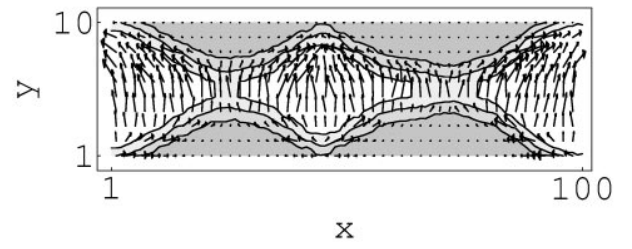

FIG. 4. (a),(b),(c) Show gradient field $\nabla h(x, y)$ and the time averaged flux of $A$ and $B$ particles on the surface are plotted by arrows with the contour plot of the mixing ratio $\rho(x, y)$, respecitvely. These quantities are averaged from $t=9000$ to $t=10000$. To see the gradient and fluxes along the axial direction $(\nabla h)_{x}$ precisely, the $x$ component of these arrows is magnified by 20 times. $f_{A A}=0.4, f_{A B}=0.44$, and $f_{B B}=1.2$. 

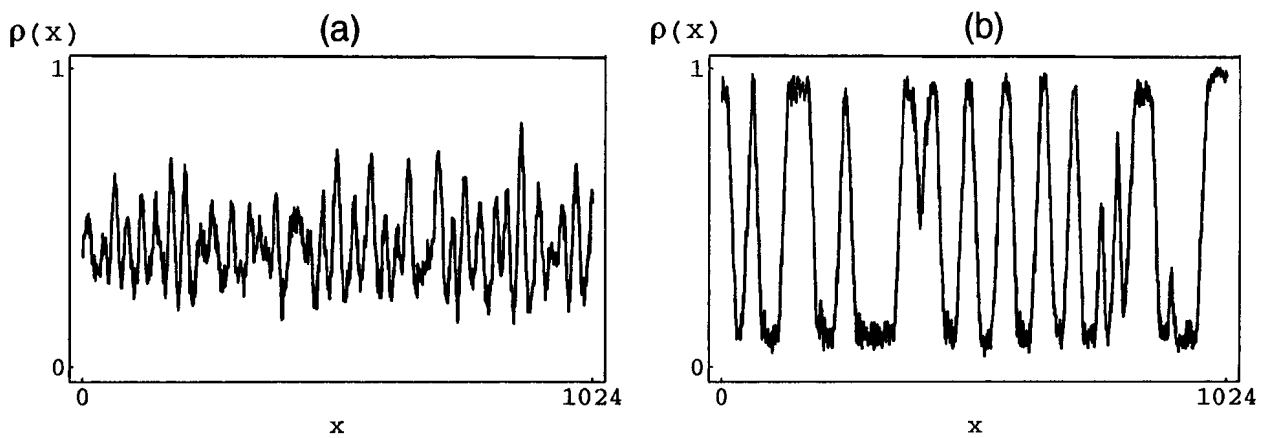

FIG. 5. The coarsening process of the axial segregation. The domain growth is shown by the mixing ratio $\rho(x)$, with (a) $t=2500$ and (b) $t=10^{5} . f_{A A}=0.4, f_{A B}=0.44$, and $f_{B B}=1.0 ; N_{x}=10, N_{y}=1024$, and $N_{z}=40$.

the axial banding. Since the time scales of the rotation and the diffusion of particles along the axial direction are quite different, the coarsening shows very slow dynamics. To see the spatiotemporal dynamics of the domain growth, the evolution of the mixing ratio $\rho(x)=$ $\sum_{y=1, z=1}^{N_{y}, N_{z}} \Theta(\sigma(x, y, z)-1) / \Theta(\sigma(x, y, z))$ is studied. In Fig. 5, snapshots of $\rho(x)$ for a typical time evolution are plotted. After calculating the power spectrum of $\rho(x)$, we can determine the characteristic wave number which takes a maxima of the power spectrum. The characteristic wave number decreased with time, indicating the coarsening process of the band pattern. Our model reproduces this slow dynamics of the segregation of the axial pattern.

In summary, we have developed a three-dimensional cellular automaton model to simulate the segregation of binary mixtures of granular materials in a rotating cylinder. Our model reproduces the transition from the radial to axial segregation. The sorting process on the flowing surface generates these segregations without considering the bulk particle motion. For axial segregation, we have found that the undulations of the core start to appear and expand into the $x=0$ front wall to form a complete banding pattern. For a smaller difference in the dynamic angle of repose $f_{A A}-f_{B B}$, these undulations did not reach the front wall during our simulation. These observations are in agreement with recent MRI experiments [15]. Furthermore, the axial segregation slows down and the wavelength of the undulation is increased. Our model is numerically efficient enough to investigate large spatiotemporal phenomenon such as the coarsening process of the band structure. It is also interesting to consider the effects of rotational speed, relative proportion, and end wall properties. These examples will be reported elsewhere.

The author would like to thank K. Ueda and R. Kobayashi for illuminating comments, and especially for the information about their experiments. I also appreciate the Particulate Science and Technology Group of the Colorado School of Mines for hosting my visit and, in particular, M. Nakagawa for his critical reading of the manuscript and useful discussions. This work is partially supported by Grant-in-Aids for Scientific Research from the Ministry of Education, Science, and Culture of Japan.

*Electronic address: http://aurora.es.hokudai.ac.jp

[1] J. C. Williams, Powder Technol. 15, 245-251 (1976).

[2] D. S. Parsons, Powder Technol. 13, 245-277 (1976).

[3] A. Rosato, K. J. Strandburg, and R. H. Swendsen, Phys. Rev. Lett. 58, 1038-1040 (1987).

[4] J. B. Knight, H. M. Jaeger, and S. R. Nagel, Phys. Rev. Lett. 70, 3278-3731 (1993).

[5] P. R. King, A. H. Makse, S. Havlin, and H.E. Stanley, Nature (London) 386, 379-382 (1997).

[6] Y. Oyama, Bull. Inst. Phys. Chem. Res. Jpn. Rep. 18, 600639 (1939) (in Japanese).

[7] M. B. Donald and B. Roseman, Br. Chem. Eng. 7, 749753 (1962).

[8] B. Roseman and M. B. Donald, Br. Chem. Eng. 7, 823827 (1962).

[9] T.C. A. Molteno, K. Choo, and S. W. Morris, Phys. Rev. Lett. 79, 2975-2978 (1997).

[10] S. S. Weidenbaum, in Advances in Chemical Engineering, edited by T.B. Drew and J.W. Hoopes (Academic, New York, 1958), Vol. 2, p. 211.

[11] S. K. Bhatia, S. Das Gupta, and D. V. Khalhar, Chem. Eng. Sci. 46, 1513-1517 (1991).

[12] L. C. Jia, P. Y. Lai, and C. K. Chan, Phys. Rev. Lett. 79, 4994-4997 (1997).

[13] D. E. Wolf, G. Baumann, and I. M. Janosi, Europhys. Lett. 27, 203-208 (1994).

[14] O. Zik et al., Phys. Rev. Lett. 73, 644-647 (1994).

[15] K. M. Hill, A. Caprihan, and J. Kakalios, Phys. Rev. Lett. 78, 50-53 (1997).

[16] M. Nakagawa et al., Chem. Eng. Sci. 52, 4423-4428 (1997).

[17] M. Nakagawa (private communication).

[18] S. Wolfram, Theory and Application of Cellular Automata (World Scientific, Singapore, 1986).

[19] P. Cizeau, A.H. Makse, and H.E. Stanley, Phys. Rev. Lett. 78, 3298-3301 (1997). 